\title{
In Vivo Effects of a Hydroxyapatite-Based Oral Care Gel on the Calcium and Phosphorus Levels of Dental Plaque
}

\author{
Henny Sudradjat ${ }^{1}$ Frederic Meyer ${ }^{2}$ Kateryna Loza \\ ${ }^{1}$ Department for Dentistry, Health Care Center, Braunschweig, \\ Germany \\ ${ }^{2}$ Research Department, Dr. Kurt Wolff GmbH \& Co. KG, Bielefeld, \\ Germany \\ ${ }^{3}$ Inorganic Chemistry and Center for Nanointegration \\ Duisburg-Essen (CeNIDE), University of Duisburg-Essen, \\ Essen, Germany
}

Matthias Epple ${ }^{3} \quad J o a c h i m ~ E n a x ²$

\begin{abstract}
Address for correspondence Joachim Enax, Research Department, Dr. Kurt Wolff GmbH \& Co. KG, Johanneswerkstr, 34-36, 33611 Bielefeld, Germany (e-mail: joachim.enax@drwolffgroup.com). Frederic Meyer, Research Department, Dr. Kurt Wolff GmbH \& Co. KG, Johanneswerkstr, 34-36, 33611 Bielefeld, Germany (e-mail: frederic.meyer@drwolffgroup.com).
\end{abstract}

\begin{abstract}
Keywords

- caries

- energy-dispersive X-ray spectroscopy

- hydroxyapatite

- oral care

- dental plaque

- calcium and phosphorus levels

Objectives Particulate hydroxyapatite (HAP; $\left.\mathrm{Ca}_{5}\left(\mathrm{PO}_{4}\right)_{3}(\mathrm{OH})\right)$ is a biomimetic oral care ingredient. One mode of action in caries-susceptible individuals may be the adhesion/ incorporation of HAP into dental plaque. Therefore, the aim of this in vivo study was to analyze the 3-day effects of a newly developed hydroxyapatite-based oral care gel on the calcium and phosphorus levels within the dental plaque of children.

Materials and Methods This study was conducted in Kebon Padangan at Bali in Indonesia. Thirty-four children (mean age, 8.9 years; mean DMF-T [decayed, missing, and filled teeth; permanent teeth], 0.6; mean dmft-t [primary teeth] 4.5) were included in the study. The gel was applied thrice for 3 days by an experienced dentist. Dental plaque was collected at baseline and after the study. Levels of calcium and phosphorus of plaque samples were analyzed by energy-dispersive X-ray spectroscopy. Statistical Analysis Medians for both calcium and phosphorus levels were calculated (baseline and 3-day application of HAP-gel).

Results The calcium level increased after 3 days of application of the HAP-gel from $0.25 \mathrm{wt} \%$ (median) to $0.40 \mathrm{wt} \%$ (median), while the phosphorus level increased from $1.17 \mathrm{wt} \%$ (median) to $1.41 \mathrm{wt} \%$ (median). However, variations in both calcium and phosphorus levels measured in the pooled dental plaque samples were high.

Conclusion Within the limitations of the study, the 3-day application of the oral HAP-gel in children increased the median of both calcium and phosphorus levels in plaque. Consequently, a positive influence on the remineralization/demineralization process is very likely.
\end{abstract}

\section{Introduction}

The presence of cariogenic dental biofilms (dental plaque) is a major factor for the development of dental caries which is a disease globally affecting millions of individuals. ${ }^{1,2}$ Preventive strategies for oral care are mainly based on (1) mechanical dental plaque removal (e.g., tooth brushing and flossing), (2) antibacterial strategies based on chemical agents (e.g., chlorhexidine, metal salts), and (3) remineralizing agents (e.g., fluorides and calcium phosphates)., ${ }^{2,3}$ Interestingly, even by dental staff (i.e., dentists, dental students, and dental assistants) it is not possible to achieve a complete plaque removal. ${ }^{4}$ Dental plaque itself has a complex composition and contains more than 700 different bacterial species. ${ }^{5}$ While most of the oral microorganisms are commensals, some of the bacteria and fungi (e.g., Candida albicans) are promoting the dental caries process. These bacteria are mainly acidic (acid producer) and aciduric (acid resistant) species from the genera Streptococcus, Lactobacillus, Prevotella, Veillonella, and others. ${ }^{2,6-8}$ Consequently, it is important to control the oral 
biofilm using mechanical and chemical approaches. However, dental biofilms can also persist interdental or in dental fissures. ${ }^{9}$ Therefore, agents are needed that can interact with dental biofilms and decrease its ability to demineralize the tooth mineral and increase the remineralization.

Due to its high similarity to human enamel crystallites, particulate hydroxyapatite ( $\mathrm{HAP}, \mathrm{Ca}_{5}\left(\mathrm{PO}_{4}\right)_{3}(\mathrm{OH})$ ) is a promising biomimetic agent in oral care formulations that is used in different products such as toothpastes, mouthwashes, and gels. ${ }^{10,11}$ HAP has an excellent biocompatibility and, in contrast to fluorides, does not lead to dental fluorosis, that is, it is ideally suited for all age groups including infants and young children. ${ }^{12}$

Its application, in any reasonable dose, does not pose any risks for a patient's health. ${ }^{13}$ HAP particles interact with enamel surfaces as shown in vitro, ${ }^{14}$ in situ, ${ }^{15}$ and in vivo. ${ }^{16,17}$ Clinical studies have shown effects of HAP-based toothpastes, for example, in prevention of enamel caries ${ }^{17}$ and reducing dentin hypersensitivity, ${ }_{1}^{18}$ as well as in improving periodontal health. ${ }^{19}$ Additionally, HAP particles reduce bacterial colonization to enamel surfaces in situ similar to chlorhexidine without having antibacterial effects, but showing antiadherent properties. ${ }^{15}$

HAP for oral care applications may differ in composition (e.g., substitutions of foreign ions for calcium like zinc ${ }^{16}$ ), as well as in crystallite morphology, and crystallite size. ${ }^{10}$ A recently published review article describes the multifunctionality of HAP and its modes of action in preventive oral health care. ${ }^{20}$ One of the described modes of action of HAP is the release of calcium and phosphate ions (i.e., a partial dissolution of HAP) after an acidic attack ( $\mathrm{pH}<5.5)$, that is, HAP may act as a calcium and phosphate reservoir when present at the tooth surface and/or in the plaque. The simplified corresponding equation is given as follows:

$$
\begin{aligned}
& \mathrm{Ca}_{5}\left(\mathrm{PO}_{4}\right)_{3}(\mathrm{OH})(\mathrm{s})+4 \mathrm{H}^{+}(\mathrm{aq}) \rightarrow 5 \mathrm{Ca}^{2+}(\mathrm{aq})+3 \mathrm{HPO}_{4}{ }^{2-}(\mathrm{aq})+ \\
& \mathrm{H}_{2} \mathrm{O}(\mathrm{l}) \\
& \quad(\mathrm{s}=\text { solid; aq = aqueous; } \mathrm{l} \text { = liquid })
\end{aligned}
$$

Acidic $\mathrm{pH}$ values are easily reached in the oral cavity both due to erosion (e.g., by soft drinks: $\mathrm{pH}$, approximately $2.5-3.5)^{21}$ and by cariogenic biofilms $(\mathrm{pH}<5.5){ }^{2}$

Shaw et al showed that plaque samples of caries-free children contain more calcium and phosphorus than plaque of caries-positive children. ${ }^{22}$ Bayrak et al also found higher calcium and phosphorus levels in plaque of caries-free individuals compared with caries-positive individuals; however, the differences were not statistically significant. ${ }^{23}$ In the field of erosion protection, the presence of calcium and phosphate ions reduces the erosive potential of acidic foods (e.g., milk or yogurt). ${ }^{24}$ Likewise, adding HAP to acidic sport drinks decreases their erosive effect. ${ }^{25}$

Thus, increasing calcium and phosphate levels at the tooth surface and/or in plaque by using calcium phosphate-based oral care products, is a promising preventive strategy to influence the demineralization/remineralization process positively to remineralization..$^{20,26-28}$

Therefore, the aim of this in vivo study was to analyze the 3-day effects of a newly developed hydroxyapatite-based oral care gel on the calcium and phosphorus levels within the dental plaque of children. To the best of the authors' knowledge, this is the first study analyzing the effects of HAP in dental plaque under in vivo conditions.

\section{Materials and Methods}

\section{General Information}

The study was registered at ClinicalTrials.gov (NCT03956992). It was conducted in Kebon Padangan, Bali, Indonesia (August 08-10, 2019) and was approved by the local health department. Parents and children gave their informed consent prior to their inclusion into the study. We chose this region because the children there have high amounts of dental plaque because daily tooth brushing is not common. Children were recruited with the help of the local school. The following inclusion and exclusion criteria were applied:

Inclusion criteria included the following:

- Children (boys and girls), 7-11 years.

- Visible dental plaque.

$-\geq 4$ teeth.

- Overall good health.

Exclusion criteria included the following:

- Allergy to one or more ingredients of the gel.

- Use of medications (e.g., antibiotics).

- Other significant reasons as decided by the principal investigator.

Case report forms were used which contained patient no., sex, date of birth (month/year), DFM-T (permanent teeth) and dmf-t (primary teeth) indices (DMF-T/dmf-t: decayed, missing, and filled teeth), inclusion and exclusion criteria, and an application diary.

In total, 34 patients ( 22 boys, 12 girls, mean age: 8.9 years; mean DMF-T: 0.6; mean dmf-t: 4.5 ) were included in the study. All patients completed the study from start to end. For each patient, baseline values (calcium and phosphorus) were used as control (no treatment) and compared with the values measured in the dental plaque samples collected at the end of the study.

\section{Composition and Application of the Hydroxyapatite Gel}

The formulation of the oral care gel was based on a newly developed gel (Karex gelée; Dr. Kurt Wolff GmbH \& Co. KG, Bielefeld, Germany), but slightly modified with the aim to exclude all calcium-based ingredients, except for the main active ingredient, that is, HAP microclusters. ${ }^{14}$ Thus, possible changes before/after the study in plaque calcium and phosphorus levels could be assigned to only one ingredient, that is, to HAP. The gel was filled in optically neutral tubes and contained the following ingredients (International Nomenclature of Cosmetic Ingredients [INCI]):

Ingredients: AQUA, HYDROXYAPATITE, GLYCERIN, HYDROGENATED STARCH HYDROLYSATE, HYDROXYETHYLCELLULOSE, PEG-40 HYDROGENATED CASTOR OIL, XYLITOL, HYDROXYACETOPHENONE, 1,2-HEXANEDIOL, CAPRYLYL GLYCOL, AROMA, 
STEVIA REBAUDIANA LEAF/STEM POWDER, PROPYLENE GLYCOL, SODIUM HYDROXIDE, LIMONENE, CITRAL.

The safety of the HAP gel was confirmed by a cosmetic product safety report according to the Regulation (EC) $1223 / 2009$ on cosmetic products before starting the study.

The gel was manually applied (finger) for 3 days and thrice daily by an experienced dentist (H.S.) using approximately $2 \mathrm{~cm}$ of the oral gel for the upper jaw and lower jaw, respectively. The gel remained in the mouth, no immediate rinsing with water was performed after the application. After application, the patients were sent home without further instructions regarding eating, drinking, and others. In general, all patients did not brush their teeth, as oral hygiene is not commonly used in this region. Dental plaque was collected with a dental excavator (sterile, for single-use only). To exclude any bias which might occur during the plaque collection, sampling altered from subject to patient (i.e., $A, B, A, B, \ldots$ ):

1. Plaque collection (baseline): quadrant one-fourth; plaque collection (after 3 days): quadrant two-thirds.

2. Plaque collection (baseline): quadrant two-thirds; plaque collection (after 3 days): quadrant one-fourth.

Plaque samples were stored in 2-mL Eppendorf tubes (DNA LoBind tubes PCR clean).

\section{Course of the study}

Day 1 (August 08, 2019):

Plaque collection (buccal and labial surfaces; no surfaces with calculus):

1. Application of the gel in the morning (after collecting of plaque).

2. Application of the gel at noon (after lunch).

3. Application of the gel in the evening (after dinner).

Day 2 (August 09, 2019):

4. Application of the gel in the morning (after breakfast).

5. Application of the gel at noon (after lunch).

6. Application of the gel in the evening (after dinner).

Day 3 (August 10, 2019):

7. Application of the gel in the morning (after breakfast).

8. Application of the gel at noon (after lunch).

9. Application of the gel in the evening (after dinner).

One hour after application of the gel: plaque collection (buccal and labial surfaces; no surfaces with calculus).

Note: 29 patients received nine applications of the gel; 1 patient received eight applications of the gel, 1 patient received seven applications of the gel, 3 patients received six applications of the gel, 5 patients missed some applications for unknown reasons; however, they received a minimum of six oral care gel applications (i.e., an average of twice daily application was ensured). Consequently, they were included in the further analysis.

\section{Analysis of Dental Plaque Samples}

Calcium and phosphorus in dental plaque samples of 29 patients were quantitatively analyzed by energydispersive X-ray spectroscopy ${ }^{29}$ (EDX; Thermo Scientific UltraDry EDS X-ray detector; in combination with a scanning electron microscope, Apreo S LoVac Thermo Scientific) at the University of Duisburg-Essen (inorganic chemistry). Note that 34 patients were included into the study. We first tried to analyze plaque samples of four randomly selected patients by atomic absorption spectroscopy (AAS) because it is a very accurate analytical technique for the quantitative analysis of calcium. However, due to insufficient quantity of plaque, we could not perform AAS analyses. Additionally, one patient was excluded from statistical analysis because of extremely high calcium values in the baseline samples (mean calcium for this patient was $20.6 \pm 2.71 \mathrm{wt} \%$ and mean calcium of all other patients was $0.74 \pm 1.82 \mathrm{wt} \%$ ). For the remaining 29 patients, we conducted three EDX analyses of plaque samples at baseline and three EDX analyses of plaque samples after the study. Afterward, the mean values were calculated for each patient (weight \%), the plaque samples were applied onto silicon single crystal substrates and dried at $60^{\circ} \mathrm{C}$ for 1 hour in air. The EDX analyses were performed at $10 \mathrm{kV}$ at a probe current of $0.9 \mathrm{nA}$. Quantitative analysis was performed by calculating the relative concentrations of chemical elements in the sample from the relative X-ray counts and applying matrix corrections. Here, a standard less iterative Phi-Rho- $Z$ matrix correction process was used for the depth distribution function (Phi), the mass density (Rho), and the mean atomic number $(Z)$, as well as for fluorescence correction. Element concentrations were determined by multiplying a measured $\mathrm{K}$-ratio by the matrix correction. The data are normalized to $100 \%$ (C, N, O, Na, Mg, K, Ca, P, S, and Cl). Overall medians for both calcium and phosphorus were calculated using the means of the 29 patients.

\section{Statistical Tests}

All means and standard deviations, as well as medians, were calculated using Microsoft Excel. To test any statistical significance of the data (baseline means versus end of study means), a two-sided $t$-test was performed using Microsoft Excel.

\section{Results and Discussion}

This study was conducted under in vivo conditions and showed that both median calcium and phosphorus levels in dental plaque samples of children increased after using the HAP-gel for 3 days (-Table 1 ). The application of HAP led to an increase of calcium and phosphorus in the dental plaque. However, the increase was not statistically significant $(p>0.05)$ but showed a tendency. Following this, the study indicates a potential interaction of HAP and the oral biofilm and/or the tooth surface (i.e., new plaque grown on HAP-coated tooth surfaces). This is in line with studies showing the interaction of HAP particles with enamel surfaces. ${ }^{14-16}$ For example, Kensche et al found an accumulation of HAP particles into the pellicle after using a HAP-based mouthwash in a transmission electron microscopic (TEM) study. ${ }^{30}$ The interaction may be based on mineral bridges between HAP particles and enamel surfaces as shown by Fabritius-Vilpoux et al in a scanning electron microscopy (SEM) study. ${ }^{14}$ The interaction of HAP particles with oral bacteria 
was also shown in different studies, for example, by Venegas et $\mathrm{al}^{31}$ and Kensche et $a{ }^{15}$ Venegas et al analyzed the adhesion of HAP to S. mutans and different Lactobacillus spp., which are prominent bacteria of dental plaque. ${ }^{2}$ Some bacteria showed adhesion to enamel surfaces, dependent on the calcium-concentration of the aqueous medium, used in this in vitro study. ${ }^{31}$ Kensche et al analyzed spit-out samples after using a HAP-mouthwash (i.e., a HAP-water-dispersion, without additives) and found an accumulation of HAP particles around bacteria by TEM. This could explain the reduction of initial bacterial colonization to enamel surfaces by using HAP-mouthwashes in situ. ${ }^{15,32}$

Our study shows that HAP may be incorporated into the oral biofilm and/or may adhere to dental plaque. The strength of the study is the in vivo setting within a highly cariogenic study population (mean DMF-T 0.6; mean dmft-t 4.5) under real live conditions. Each patient served as its own control: biologically occurring differences, that is, in saliva composition, salivary flow, and others can be excluded. All patients used only the HAP gel for oral hygiene instructions. Additionally, the diet was nearly the same with all patients during the study period. The application of the gel was standardized as it was performed by only one experienced dentist. Thus, variations of the applications can be excluded. Sampling was also performed by the same dentist at the beginning and at the end of the study. To exclude weakly attached HAP, collection was performed 1 hour after the last gel application.

It is well known that atomic absorption spectroscopy (AAS) is the analytical method to measure calcium at high accuracy. However, this method requires a minimum amount of dry mass from a biologic sample. This amount could not be reached within this study. Therefore, EDX-analyses were used to measure the quantity of calcium and phosphorus in the samples. EDX only needs small amounts as it is coupled to SEM. However, it only gives semiquantitative data unless the samples are polished and calibration standards are used. ${ }^{29}$ Other studies quantified calcium and phosphorus in plaque samples by $\operatorname{AAS}^{22,33}(\mathrm{Ca})$, ion chromatography ${ }^{23}$ ( $\mathrm{Ca}$ and phosphate), or by microcolorimeter ${ }^{34}$ (Ca and phosphate). Further suitable methods are inductively coupled plasma mass spectrometry and X-ray fluorescence spectroscopy.

Nevertheless, the calcium and phosphorus content is comparable to results reported by Shaw et al, ${ }^{22}$ they analyzed dental plaque samples of 23 caries-free and 32 caries-active children and found calcium contents for caries-active children (posterior plaque: $1.63 \mu \mathrm{g} / \mathrm{mg} \bumpeq 0.163 \mathrm{wt} \%$ and anterior plaque: $2.57 \mu \mathrm{g} / \mathrm{mg} \triangleq 0.257 \mathrm{wt} \%$ ). Although we did not differentiate between anterior and posterior plaque, these values are comparable to our EDX-results ( - Table $\mathbf{1}$ ).

Furthermore, Shaw et al reported calcium levels of caries-free children (posterior plaque: $3.57 \mu \mathrm{g} / \mathrm{mg} \cong 0.357 \mathrm{wt} \%$ and anterior plaque: $11.55 \mu \mathrm{g} / \mathrm{mg} \bumpeq 1.155 \mathrm{wt} \%$ ). These results clearly show that a naturally (i.e., by saliva) increased calcium content of the dental plaque is correlated with a decreased rate of caries. ${ }^{22}$ It appears reasonable that by regularly using a HAP-based gel, the calcium content of the dental plaque may be artificially increased.
Table 1 Calcium and phosphorus levels of dental plaque at baseline and after 3 days application of the HAP-gel in vivo (29 children)

\begin{tabular}{|l|l|l|}
\hline EDX analyses & Baseline & After the study \\
\hline Calcium & Median: 0.25 wt\% & Median: 0.40 wt\% \\
& Mean with SD: 0.74 \pm & Mean with SD: 0.54 \pm \\
& 1.82 wt\% & 0.49 wt\% \\
\hline Phosphorus & Median: 1.17 wt\% & Median: 1.41 wt\% \\
& Mean with SD: $1.25 \pm$ & Mean with SD: $1.43 \pm$ \\
& 0.75 wt\% & 0.58 wt\% \\
\hline
\end{tabular}

Abbreviations: EDX, energy-dispersive X-ray; HAP, particulate hydroxyapatite; SD, standard deviation.

The variation in our data was high as shown in - Table 1 which was also reported by Shaw et al (e.g., calcium [anterior plaque]: $1.8-36.5 \mu \mathrm{g} / \mathrm{mg}$ ). These big variations can be explained by a high heterogeneity between the individual patients.

HAP was the only calcium source of the gel. Therefore, it is likely that the increased calcium content is due to an incorporation of HAP into the oral biofilm.

Zhang et al showed in an in vitro pH-cycling study that the calcium level in S. mutans biofilms treated with HAP was approximately eight-times higher than in a group treated with sodium fluoride (NaF) This resulted in an enhanced enamel demineralization protection as shown by transversal microradiography. However, no enamel remineralization was observed. ${ }^{33}$ In contrast, other in vitro and in situ studies have shown a remineralizing effect of HAP-containing toothpastes on enamel and/or dentin surfaces. Recent studies were published by Tschoppe et $\mathrm{al}^{27}$ (in vitro study), Najibfard et al, ${ }^{26}$ and Amaechi et $\mathrm{a}^{28}$ (the latter are in situ studies).

Besides the release of calcium and phosphate ions (i.e., remineralization and minimizing demineralization), HAP may also act as a buffer of organic acids in cariogenic biofilms. ${ }^{17,35}$ Nedeljkovic et al analyzed the acid-buffering properties of different tooth restoration materials in vitro. HAP and amalgam showed superior acid-buffering properties, compared with a glass-ionomer cement and giomer (composite with surface prereacted glass ionomer fillers; lower buffering capacity) and a conventional composite (no buffering capacity). The authors concluded that restorative materials without buffering properties increase the risk of shifting plaque composition to more cariogenic bacteria. ${ }^{35}$ The buffering effect of HAP can be explained by the release of (hydrogen/dihydrogen) phosphates after an acidic attack which may act similar to the phosphate buffer system present in saliva. ${ }^{2}$

Due to the explorative character of this pilot study, limitations are that only gel with HAP was used but no placebo gel or control gel. Additionally, patients were not under observation after gel applications. Thus, it was not possible to monitor their food intake. Finally, future studies should focus on a longer study period and a larger number of patients.

This first study clearly emphasizes the need for further studies to investigate the protective properties of biomimetic HAP acting as calcium and phosphorus reservoir for remineralization of initial carious lesions and its possible buffering potential. 
This is relevant, for example, for individuals with a high caries risk, such as orthodontic patients (high plaque values), ${ }^{17}$ and for children, as well as for individuals, with a reduced salivary flow (hyposalivation) because these individuals have a lack of calcium and phosphate ions in the oral cavity. ${ }^{36}$

Besides hydroxyapatite, the administration of other calcium phosphate minerals has led to increased calcium and phosphate levels in dental plaque. For example, Vogel et al reported on a chewing gum which contained $\alpha$-tricalcium phosphate, $\alpha-\mathrm{Ca}_{3}\left(\mathrm{PO}_{4}\right)_{2}$ (which is also a calcium phosphate mineral) and its effects on the composition of plaque and saliva after sucrose ingestion. They found higher calcium and phosphate concentrations in plaque fluid and saliva compared with a control chewing gum. ${ }^{34}$ Additionally, a paste containing CPP-ACP (casein phosphopeptides and amorphous calcium phosphate; $\left.\mathrm{Ca}_{x}\left(\mathrm{PO}_{4}\right)_{y} \cdot n \mathrm{H}_{2} \mathrm{O}\right)$ increased the calcium and phosphate levels in both saliva and plaque. ${ }^{37}$ In general, the various structures of calcium phosphates, used in oral care products, may have different efficacies. ${ }^{38} \mathrm{~A}$ systematic review and meta-analysis show that HAP reduces dentin hypersensitivity while ACP does not seem to be effective. ${ }^{18}$

\section{Conclusion}

Calcium phosphates (e.g., hydroxyapatite, $\alpha$-tricalcium phosphate, and amorphous calcium phosphate) are promising agents to increase the calcium and phosphate levels in dental plaque. ${ }^{33,34,37,38}$

Due to the high biocompatibility of HAP and the absence of any fluorosis risk for children, ${ }^{12,17}$ increasing HAP dosing by the combination of different HAP-based oral care products (such as HAP-toothpaste, HAP-mouthwash, or HAP-gel) may further increase calcium and phosphorus levels in dental plaque and consequently HAP's efficacy in preventive oral care. ${ }^{14,17}$

To conclude, within the limitations of this study, it was shown for the first time that a 3-day administration of an oral HAP-gel in a group of children (mean age, 8.9 years; mean DMF-T, 0.6; mean dmft-t, 4.5) increased medians of both calcium and phosphorus levels in plaque. Thus, a positive influence on the remineralization/demineralization process is very likely..$^{20,26-28}$

\section{Funding}

The study was funded by Dr. Kurt Wolff GmbH \& Co. KG, Bielefeld, Germany.

\section{Conflict of Interest}

None declared.

\section{Acknowledgments}

The authors would like to thank Barbara Simader for helpful discussions.

\section{References}

1 Listl S, Galloway J, Mossey PA, Marcenes W. Global economic impact of dental diseases. J Dent Res 2015;94(10):1355-1361

2 Fejerskov O, Kidd E, eds, In: Dental Caries: The Disease and Its Clinical Management. Oxford, United Kingdom: Blackwell Munksgaard Ltd; 2009

3 Loveren Cv, Toothpastes. Vol. 23. Basel, Switzerland: Karger; 2013
4 Deinzer R, Schmidt R, Harnacke D, et al. Finding an upper limit of what might be achievable by patients: oral cleanliness in dental professionals after self-performed manual oral hygiene. Clin Oral Investig 2018;22(2):839-846

5 Dewhirst FE, Chen T, Izard J, et al. The human oral microbiome. J Bacteriol 2010;192(19):5002-5017

6 Sztajer H, Szafranski SP, Tomasch J, et al. Cross-feeding and interkingdom communication in dual-species biofilms of Streptococcus mutans and Candida albicans. ISME J 2014;8(11):2256-2271

7 Teng F, Yang F, Huang S, et al. Prediction of early childhood caries via spatial-temporal variations of oral microbiota. Cell Host Microbe 2015;18(3):296-306

8 Hurley E, Barrett MPJ, Kinirons M, et al. Comparison of the salivary and dentinal microbiome of children with severe-early childhood caries to the salivary microbiome of caries-free children. BMC Oral Health 2019;19(1):13-13

9 Worthington HV, MacDonald L, Poklepovic Pericic T, et al. Home use of interdental cleaning devices, in addition to toothbrushing, for preventing and controlling periodontal diseases and dental caries. Cochrane Database Syst Rev 2019;4:CD012018

10 Enax J, Epple M. Synthetic hydroxyapatite as a biomimetic oral care agent. Oral Health Prev Dent 2018;16(1):7-19

11 Meyer F, Enax J. Hydroxyapatite in oral biofilm management. Eur J Dent 2019;13(2):287-290

12 Meyer F, Enax J. Early childhood caries: epidemiology, aetiology, and prevention. Int J Dent 2018;2018:1415873

13 Epple M. Review of potential health risks associated with nanoscopic calcium phosphate. Acta Biomater 2018;77:1-14

14 Fabritius-Vilpoux K, Enax J, Herbig M, Raabe D, Fabritius $\mathrm{H}-\mathrm{O}$. Quantitative affinity parameters of synthetic hydroxyapatite and enamel surfacesin vitro. Bioinspir Biomim Nan. 2019;8:141-153

15 Kensche A, Holder C, Basche S, Tahan N, Hannig C, Hannig M. Efficacy of a mouthrinse based on hydroxyapatite to reduce initial bacterial colonisation in situ. Arch Oral Biol 2017;80:18-26

16 Lelli M, Putignano A, Marchetti M, et al. Remineralization and repair of enamel surface by biomimetic Zn-carbonate hydroxyapatite containing toothpaste: a comparative in vivo study. Front Physiol 2014;5:333

17 Schlagenhauf U, Kunzelmann KH, Hannig C, et al. Impact of a non-fluoridated microcrystalline hydroxyapatite dentifrice on enamel caries progression in highly caries-susceptible orthodontic patients: a randomized, controlled 6-month trial. J Investig Clin Dent 2019;10(2):e12399

18 Hu ML, Zheng G, Zhang YD, Yan X, Li XC, Lin H. Effect of desensitizing toothpastes on dentine hypersensitivity: a systematic review and meta-analysis. J Dent 2018;75:12-21

19 Hagenfeld D, Prior K, Harks I, et al. No differences in microbiome changes between anti-adhesive and antibacterial ingredients in toothpastes during periodontal therapy. J Periodontal Res 2019;54(4):435-443

20 Enax J, Fabritius H-O, Fabritius-Vilpoux K, Amaechi BT, Meyer F. Modes of action and clinical efficacy of particulate hydroxyapatite in preventive oral health care - state of the art. Open Dent J 2019;13:274-287

21 Lussi A, Carvalho TS. The future of fluorides and other protective agents in erosion prevention. Caries Res 2015;49(Suppl 1): 18-29

22 Shaw L, Murray JJ, Burchell CK, Best JS. Calcium and phosphorus content of plaque and saliva in relation to dental caries. Caries Res 1983;17(6):543-548

23 Bayrak S, Okte Z, Fidanci UR. Relationship between caries and dental plaque composition. Am J Dent 2011;24(1):45-48

24 Epple M, Enax J. Moderne Zahnpflege aus chemischer Sicht. Chem Unserer Zeit 2018;52:218-228

25 Min JH, Kwon HK, Kim BI. The addition of nano-sized hydroxyapatite to a sports drink to inhibit dental erosion: in vitro study using bovine enamel. J Dent 2011;39(9):629-635 
26 Najibfard K, Ramalingam K, Chedjieu I, Amaechi BT. Remineralization of early caries by a nano-hydroxyapatite dentifrice. J Clin Dent 2011;22(5):139-143

27 Tschoppe P, Zandim DL, Martus P, Kielbassa AM. Enamel and dentine remineralization by nano-hydroxyapatite toothpastes. J Dent 2011;39(6):430-437

28 Amaechi BT, AbdulAzees PA, Alshareif DO, et al. Comparative efficacy of a hydroxyapatite and a fluoride toothpaste for prevention and remineralization of dental caries in children. BDJ Open 2019;5:18

29 Scimeca M, Bischetti S, Lamsira HK, Bonfiglio R, Bonanno E. Energy dispersive X-ray (EDX) microanalysis: A powerful tool in biomedical research and diagnosis. Eur J Histochem 2018;62(1):2841

30 Kensche A, Pötschke S, Hannig C, Richter G, Hoth-Hannig W, Hannig M. Influence of calcium phosphate and apatite containing products on enamel erosion. ScientificWorldJournal 2016;2016:7959273

31 Venegas SC, Palacios JM, Apella MC, Morando PJ, Blesa MA. Calcium modulates interactions between bacteria and hydroxyapatite. J Dent Res 2006;85(12):1124-1128

32 Hannig C, Basche S, Burghardt T, Al-Ahmad A, Hannig M. Influence of a mouthwash containing hydroxyapatite microclusters on bacterial adherence in situ. Clin Oral Investig 2013;17(3):805-814
33 Zhang M, He LB, Exterkate RA, et al. Biofilm layers affect the treatment outcomes of NaF and Nano-hydroxyapatite. J Dent Res 2015;94(4):602-607

34 Vogel GL, Zhang Z, Carey CM, Ly A, Chow LC, Proskin HM. Composition of plaque and saliva following a sucrose challenge and use of an alpha-tricalcium-phosphate-containing chewing gum. J Dent Res 1998;77(3):518-524

35 Nedeljkovic I, De Munck J, Slomka V, Van Meerbeek B, Teughels W, Van Landuyt KL. Lack of buffering by composites promotes shift to more cariogenic bacteria. J Dent Res 2016;95(8):875-881

36 Papas A, Russell D, Singh M, Kent R, Triol C, Winston A. Caries clinical trial of a remineralising toothpaste in radiation patients. Gerodontology 2008;25(2):76-88

$37 \mathrm{Hr}$ P, Ra H, Re H, H S, P P. Concentration of calcium, phosphate and fluoride ions in microbial plaque and saliva after using CPP-ACP paste in 6-9 year-old children. J Dent Biomater 2016;3(2):214-219

38 Meyer F, Amaechi BT, Fabritius HO, Enax J. Overview of calcium phosphates used in biomimetic oral care. Open Dent J 2018;12:406-423 